\title{
Comparative study of the production of scalar and tensor mesons in $e^{+} e^{-}$collisions
}

\author{
N.N. Achasov ${ }^{a}$, A.I. Goncharenko ${ }^{a, b}$, A.V. Kiselev ${ }^{a, b}$, and E.V. Rogozina ${ }^{a, b}$ \\ ${ }^{a}$ Laboratory of Theoretical Physics, Sobolev Institute \\ for Mathematics, 630090, Novosibirsk, Russia \\ ${ }^{b}$ Novosibirsk State University, 630090, Novosibirsk, Russia
}

(Dated: October 31, 2018)

\begin{abstract}
The intensity of scalar $a_{0}(980), f_{0}(980)$ and tensor $a_{2}(1320), f_{2}(1270)$ mesons production at VEPP-2000 (BINP, Novosibirsk) and the upgraded DA $\Phi$ NE (Frascati, Italy) in the processes $e^{+} e^{-} \rightarrow a_{0}\left(f_{0}, a_{2}, f_{2}\right) \gamma$ is calculated. The calculation was made with the help of VDM and the kaon loop model (for scalar mesons case). Note that the processes $e^{+} e^{-} \rightarrow a_{2}\left(f_{2}\right) \gamma$ have not been studied in the energy region of VEPP-2000.

It turned out that in the VEPP-2000 energy region $1.7-2.0 \mathrm{GeV} \sigma\left(e^{+} e^{-} \rightarrow a_{2}\left(f_{2}\right) \gamma\right) \sim 10 \mathrm{pb}$, and $\sigma\left(e^{+} e^{-} \rightarrow a_{0}\left(f_{0}\right) \gamma\right) \sim 0.1 \mathrm{pb}$.

Photon angle distribution and the spin density matrices of $a_{2}$ and $f_{2}$ were calculated also.
\end{abstract}

PACS numbers: 12.39.-x 13.40.Hq 13.66.Bc 


\section{INTRODUCTION}

Study of the nature of light scalar resonances is one of the central problems of nonperturbative QCD, it is important for understanding the chiral symmetry realization way in the low energy region and, consequently, the confinement physics. The $a_{2}(1320)$ and $f_{2}(1270)$ tensor mesons are well-known P-wave $q \bar{q}$ states. Naively one might think that the scalar $a_{0}(980)$ and $f_{0}(980)$ mesons are also the $q \bar{q}$ P-wave states with the same quark structure, as $a_{2}(1320)$ and $f_{2}(1270)$, respectively. But now there are many indications that the above scalars are the four quark states.

Comparative study of the production of scalar and tensor mesons is proposed to investigate the nature of light scalar mesons. For this purpose the intensity of scalar $a_{0}(980)$, $f_{0}(980)$ and tensor $a_{2}(1320), f_{2}(1270)$ mesons production at the colliders VEPP-2000 (BINP, Novosibirsk) and DA $\Phi$ NE (LNF, Frascati) in the processes $e^{+} e^{-} \rightarrow S \gamma, T \gamma$ (here and hereafter $\left.S=a_{0}, f_{0} ; T=a_{2}, f_{2}\right)$ is calculated.

\section{THE REACTIONS $e^{+} e^{-} \rightarrow a_{0} \gamma \rightarrow \eta \pi^{0} \gamma$ AND $e^{+} e^{-} \rightarrow f_{0} \gamma \rightarrow \pi^{0} \pi^{0} \gamma$}

As it is known, the kaon loop model [1, 2] describe the $V \rightarrow K^{+} K^{-} \rightarrow a_{0}\left(f_{0}\right) \gamma$ decays well [3 11]. The signal contribution is $e^{+} e^{-} \rightarrow \sum_{V} V \rightarrow K^{+} K^{-} \rightarrow a_{0}\left(f_{0}\right) \gamma \rightarrow \eta\left(\pi^{0}\right) \pi^{0} \gamma$, where $V$ are vector mesons. The signal cross-sections

$$
\begin{gathered}
\frac{d \sigma\left(e^{+} e^{-} \rightarrow a_{0} \gamma \rightarrow \eta \pi^{0} \gamma\right)}{d m}=\frac{g_{a_{0} \eta \pi^{0}}^{2} p_{\eta \pi}(m)}{4 \pi^{2}\left|D_{a_{0}}(m)\right|^{2}} \sigma\left(e^{+} e^{-} \rightarrow a_{0} \gamma, m\right)= \\
\frac{e^{2}\left(s-m^{2}\right) p_{\eta \pi}(m)}{96 \pi^{3} s^{3}}\left|A_{K^{+} K^{-}}(s) \bar{g}(m)\right|^{2}\left|\frac{g_{a_{0} K^{+} K^{-} g_{a_{0} \eta \pi^{0}}}}{D_{a_{0}}(m)}\right|^{2} \\
\frac{d \sigma\left(e^{+} e^{-} \rightarrow f_{0} \gamma \rightarrow \pi^{0} \pi^{0} \gamma\right)}{d m}=\frac{g_{f_{0} \pi^{0} \pi^{0}} p_{\pi \pi}(m)}{4 \pi^{2}\left|D_{f_{0}}(m)\right|^{2}} \sigma\left(e^{+} e^{-} \rightarrow f_{0} \gamma, m\right)= \\
\frac{e^{2}\left(s-m^{2}\right) p_{\pi \pi}(m)}{96 \pi^{3} s^{3}}\left|A_{K^{+} K^{-}}(s) \bar{g}(m)\right|^{2}\left|\frac{g_{f_{0} K^{+} K^{-}} g_{f_{0} \pi^{0} \pi^{0}}}{D_{f_{0}}(m)}\right|^{2}, \\
p_{\eta \pi}(m)=\frac{\sqrt{\left(m^{2}-\left(m_{\eta}+m_{\pi}\right)^{2}\right)\left(m^{2}-\left(m_{\eta}-m_{\pi}\right)^{2}\right)}}{2 m}, \quad p_{\pi \pi}(m)=\frac{\sqrt{m^{2}-4 m_{\pi}^{2}}}{2}
\end{gathered}
$$

The $A_{K^{+} K^{-}}(s)$ is the amplitude of the $\gamma^{*} \rightarrow K^{+} K^{-}$transition. Without mixing of the intermediate vector states this amplitude is 


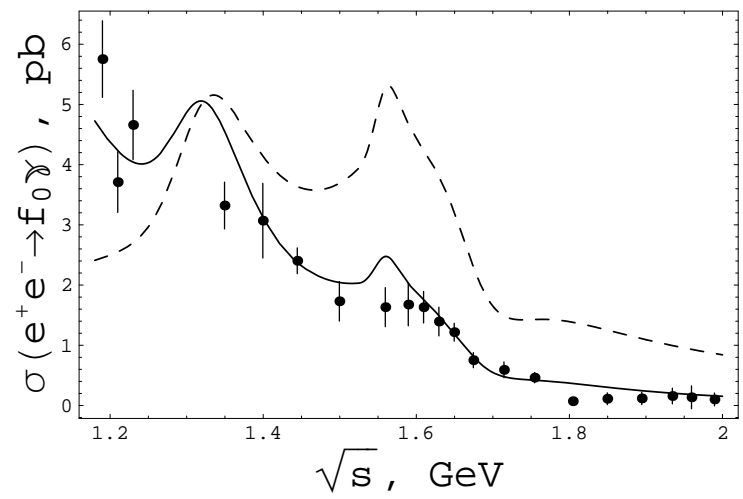

(a)

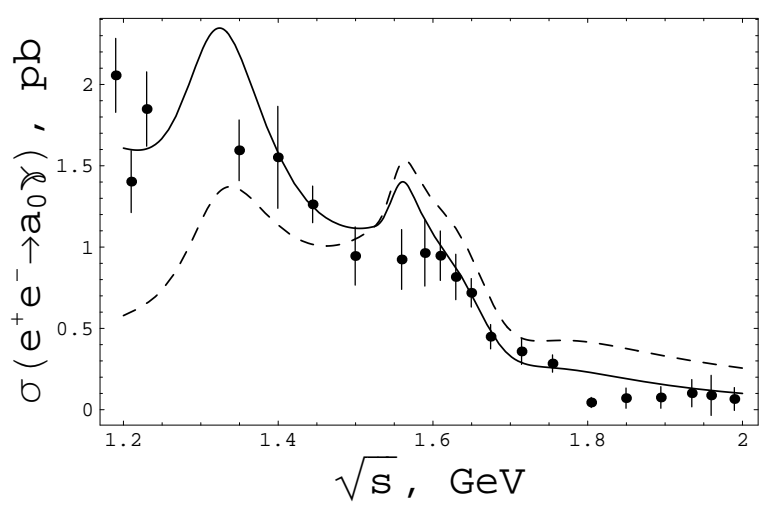

(b)

FIG. 1: The a) $\sigma\left(e^{+} e^{-} \rightarrow f_{0} \gamma\right)$ and b) $\sigma\left(e^{+} e^{-} \rightarrow a_{0} \gamma\right)$. Solid lines show the kaon loop model prediction, dashed lines show 1/10 of the cross-sections in case of the mere VDM prediction for the point-like $V S \gamma$ interaction, normalized on the signal at $\sqrt{s}=m_{\phi}$. Points are result of using the experimental data [12, 13] on the $e^{+} e^{-} \rightarrow K^{+} K^{-}$in the Eqs. (41), (5).

$$
A_{K^{+} K^{-}}(s)=\sum_{V=\rho, \rho^{\prime}, \rho^{\prime \prime}, \omega, \omega^{\prime}, \omega^{\prime \prime}, \phi, \phi^{\prime}, \phi^{\prime \prime}} \frac{g_{V \gamma} g_{V K^{+} K^{-}}}{D_{V}(s)}
$$

We took into account mixing of the resonances according to Ref. [14]. The $m$ is the $\eta \pi^{0}$ or $\pi^{0} \pi^{0}$ invariant mass correspondingly, $g_{f_{0} \pi^{0} \pi^{0}}=g_{f_{0} \pi^{+} \pi^{-}} / \sqrt{2}$ for the pions identity and $\bar{g}(m)=\frac{g_{R}(m)}{g_{\phi K^{+} K^{-}} g_{R K^{+} K^{-}}}$is the integral on the kaon loop [1]. The $D_{a_{0}}(m)$ and $D_{f_{0}}(m)$ are the inverse propagators of scalar mesons, they are taken from [9] and [11] (2012 paper) correspondingly. All parameters of the $a_{0}(980)$ and $f_{0}(980)$ are taken from [9] (Fit 1) and [11] (Fit 1 of the 2012 paper) correspondingly. The Eqs. (11) and (2) may be rewritten as

$$
\begin{aligned}
\frac{d \sigma\left(e^{+} e^{-} \rightarrow a_{0} \gamma \rightarrow \eta \pi^{0} \gamma\right)}{d m}= & \frac{\left(s-m^{2}\right) p_{\eta \pi}(m)}{2 \pi^{2} \sqrt{s}\left(s-4 m_{K^{+}}^{2}\right)^{3 / 2}}|\bar{g}(m)|^{2}\left|\frac{g_{a_{0} K^{+} K^{-}} g_{a_{0} \eta \pi^{0}}}{D_{a_{0}}(m)}\right|^{2} \times \\
& \sigma\left(e^{+} e^{-} \rightarrow K^{+} K^{-}\right), \\
\frac{d \sigma\left(e^{+} e^{-} \rightarrow f_{0} \gamma \rightarrow \pi^{0} \pi^{0} \gamma\right)}{d m}= & \frac{\left(s-m^{2}\right) p_{\pi \pi}(m)}{2 \pi^{2} \sqrt{s}\left(s-4 m_{K^{+}}^{2}\right)^{3 / 2}}|\bar{g}(m)|^{2}\left|\frac{g_{f_{0} K^{+} K^{-}} g_{f_{0} \pi^{0} \pi^{0}}}{D_{f_{0}}(m)}\right|^{2} \times \\
& \sigma\left(e^{+} e^{-} \rightarrow K^{+} K^{-}\right)
\end{aligned}
$$

The photon angular distribution is 


$$
\frac{d n_{\gamma}^{S}}{d \Omega}=\frac{3}{16 \pi}\left(1+\cos ^{2} \theta\right),
$$

where $\theta$ is the angle between the $\gamma$ momentum and the beam axis.

In Fig. 11 we show the $\sigma\left(e^{+} e^{-} \rightarrow K^{+} K^{-} \rightarrow S \gamma\right)$ cross-sections prediction, the mere VDM one (point-like $V S \gamma$ interaction) is shown also [15]. Points on Fig. 1 are obtained with the help of the experimental data [12, 13] on the $e^{+} e^{-} \rightarrow K^{+} K^{-}$and Eqs. (44), (5). Note that $\sigma\left(e^{+} e^{-} \rightarrow a_{0} \gamma \rightarrow \eta \pi^{0} \gamma\right)$ and $\sigma\left(e^{+} e^{-} \rightarrow f_{0} \gamma \rightarrow \pi^{0} \pi^{0} \gamma\right)$ are considerably less for the branching fractions and the energy cut of the narrow $a_{0}$ and $f_{0}$ resonance regions.

Note also that the Vector Dominance Model describes formfactors (and transition formfactors) of $q \bar{q}$ states in the low and intermediate energy regions. In case of the four quark states $\mathrm{S}=a_{0}, f_{0}$ the amplitude of the process $\gamma^{*} \rightarrow S \gamma$ along with VDM suppression $\left(m_{Y}^{2}-s\right)^{-1}$ has also additional suppression $\frac{\left(2 m_{K}\right)^{2}}{s} \ln ^{2} \frac{s}{m_{K}^{2}}$ with increasing $s$ for the kaon loop [16]. This provides additional suppression in comparison with the $q \bar{q}$ state case, see Fig. 11.

\section{THE REACTION $e^{+} e^{-} \rightarrow a_{2}\left(f_{2}\right) \gamma$}

It is known that in the reaction $\gamma \gamma \rightarrow f_{2} \rightarrow \pi \pi$ tenzor mesons are produced mainly by the photons with the opposite helicity states. The effective Lagrangian in this case is

$$
\begin{gathered}
L=g_{f_{2} \gamma \gamma} T_{\mu \nu} F_{\mu \sigma} F_{\nu \sigma} \\
F_{\mu \sigma}=\partial_{\mu} A_{\sigma}-\partial_{\sigma} A_{\mu}
\end{gathered}
$$

where $A_{\mu}$ is a photon field and $T_{\mu \nu}$ is a tenzor $f_{2}$ field. So in the frame of Vector Dominance Model we assume that the effective Lagrangian of the reaction $f_{2} \rightarrow V V$ is [17]:

$$
\begin{gathered}
L=g_{f_{2} V V} T_{\mu \nu} F_{\mu \sigma}^{V} F_{\nu \sigma}^{V}, \\
F_{\mu \sigma}^{V}=\partial_{\mu} V_{\sigma}-\partial_{\sigma} V_{\mu} .
\end{gathered}
$$

where $V=\rho, \rho^{\prime}, \rho^{\prime \prime} \omega, \omega^{\prime}, \omega^{\prime \prime}$. Note that $\omega, \omega^{\prime}$ and $\omega^{\prime \prime}$ give $\sim 10 \%$ of the $\rho, \rho^{\prime}$ and $\rho^{\prime \prime}$ contribution in the amplitude, so then we neglect them: our current aim is to obtain estimates only. The $\rho-\rho^{\prime}-\rho^{\prime \prime}$ mixing is omitted here also.

Assuming the VDM mechanism $e^{+} e^{-} \rightarrow\left(\rho+\rho^{\prime}+\rho^{\prime \prime}\right) \rightarrow f_{2}\left(\rho+\rho^{\prime}+\rho^{\prime \prime}\right) \rightarrow f_{2} \gamma$ one obtains 


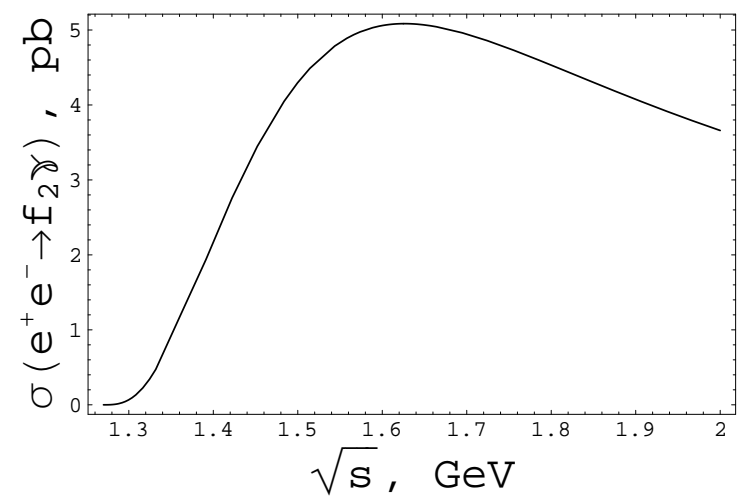

(a)

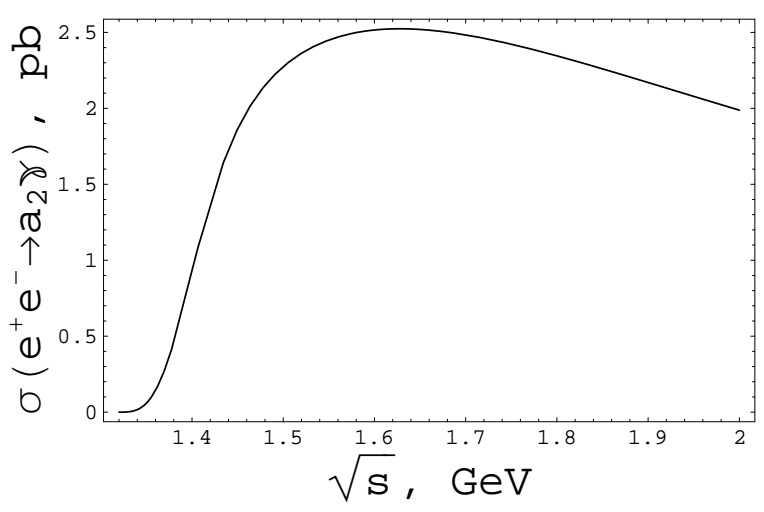

(b)

FIG. 2: a) The $\sigma\left(e^{+} e^{-} \rightarrow f_{2} \gamma\right)$ for $g_{f_{2} \rho^{\prime \prime} \rho^{\prime \prime}}=g_{f_{2} \rho^{\prime} \rho^{\prime}}=g_{f_{2} \rho \rho}$. b) The $\sigma\left(e^{+} e^{-} \rightarrow a_{2} \gamma\right)$ for $g_{a_{2} \rho^{\prime \prime} \omega^{\prime \prime}}=$ $g_{a_{2} \rho^{\prime} \omega^{\prime}}=g_{a_{2} \rho \omega}$.

$$
\begin{gathered}
\sigma\left(e^{+} e^{-} \rightarrow f_{2} \gamma\right)=\frac{4 \pi^{2}}{9} \alpha^{3}\left(1-\frac{m_{f_{2}}^{2}}{s}\right)^{3}\left(\frac{s^{2}}{m_{f_{2}}^{4}}+3 \frac{s}{m_{f_{2}}^{2}}+6\right) \times \\
\left|\frac{m_{\rho}^{2} g_{f_{2} \rho \rho}}{f_{\rho}^{2} D_{\rho}(s)}+\frac{m_{\rho^{\prime}}^{2} g_{f_{2} \rho^{\prime} \rho^{\prime}}}{f_{\rho^{\prime}}^{2} D_{\rho^{\prime}}(s)}+\frac{m_{\rho^{\prime \prime}}^{2} g_{f_{2} \rho^{\prime \prime} \rho^{\prime \prime}}}{f_{\rho^{\prime \prime}}^{2} D_{\rho^{\prime \prime}}(s)}\right|^{2}
\end{gathered}
$$

and

$$
\Gamma\left(f_{2} \rightarrow \gamma \gamma\right)=\frac{\pi \alpha^{2}}{5}\left|\frac{g_{f_{2} \rho \rho}}{f_{\rho}^{2}}+\frac{g_{f_{2} \rho^{\prime} \rho^{\prime}}}{f_{\rho^{\prime}}^{2}}+\frac{g_{f_{2} \rho^{\prime \prime} \rho^{\prime \prime}}}{f_{\rho^{\prime \prime}}^{2}}\right|^{2} m_{f_{2}}^{3}=3.03 \pm 0.35 \mathrm{keV}[18] \text {. }
$$

We use the same parameters of the $\rho, \rho^{\prime}, \rho^{\prime \prime}$ as for the $e^{+} e^{-} \rightarrow a_{0} \gamma$ and $e^{+} e^{-} \rightarrow f_{0} \gamma$ reactions. It is assumed that $g_{f_{2} \rho \rho^{\prime}}$ and other cross constants are suppressed due to small overlap of the spatial wave functions of $\rho, \rho^{\prime}$ and $\rho^{\prime \prime}$. The $f_{V}$ is obtained from the relation

$$
\Gamma\left(V \rightarrow e^{+} e^{-}, s\right)=\frac{4 \pi \alpha^{2}}{3 f_{V}^{2}} \frac{m_{V}^{4}}{s^{3 / 2}}
$$

If one assumes that $g_{f_{2} \rho \rho}=g_{f_{2} \rho^{\prime} \rho^{\prime}}=g_{f_{2} \rho^{\prime \prime} \rho^{\prime \prime}}$, then the Fig. 2a is obtained.

The $\pi^{0} \pi^{0}$ invariant mass $m$ spectra is

$$
\begin{gathered}
\frac{d \sigma\left(e^{+} e^{-} \rightarrow f_{2} \gamma \rightarrow \pi^{0} \pi^{0} \gamma\right)}{d m}=\frac{8 \pi}{9} \alpha^{3}\left(1-\frac{m^{2}}{s}\right)^{3}\left(\frac{s^{2}}{m_{f_{2}}^{4}}+3 \frac{s}{m_{f_{2}}^{2}}+6\right) \times \\
\left|\frac{m_{\rho}^{2} g_{f_{2} \rho \rho}}{f_{\rho}^{2} D_{\rho}(s)}+\frac{m_{\rho^{\prime}}^{2} g_{f_{2} \rho^{\prime} \rho^{\prime}}}{f_{\rho^{\prime}}^{2} D_{\rho^{\prime}}(s)}+\frac{m_{\rho^{\prime \prime}}^{2} g_{f_{2} \rho^{\prime \prime} \rho^{\prime \prime}}}{f_{\rho^{\prime \prime}}^{2} D_{\rho^{\prime \prime}}(s)}\right|^{2} \frac{\Gamma\left(f_{2} \rightarrow \pi^{0} \pi^{0}, m\right) m^{2}}{\left|D_{f_{2}}(m)\right|^{2}}
\end{gathered}
$$




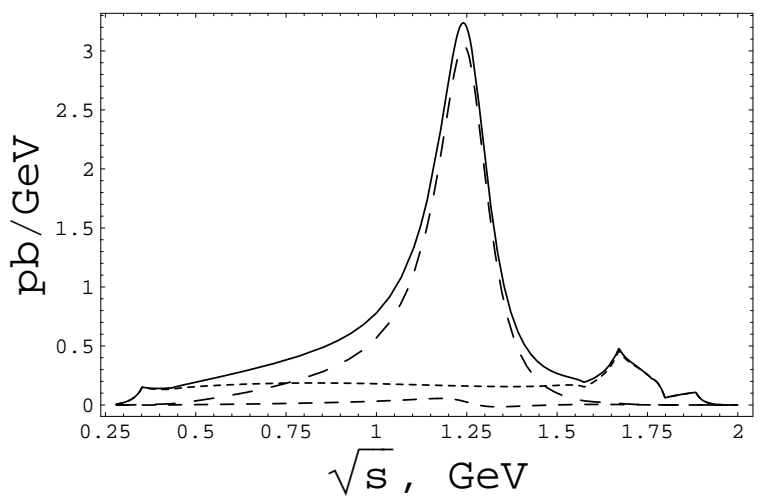

(a)

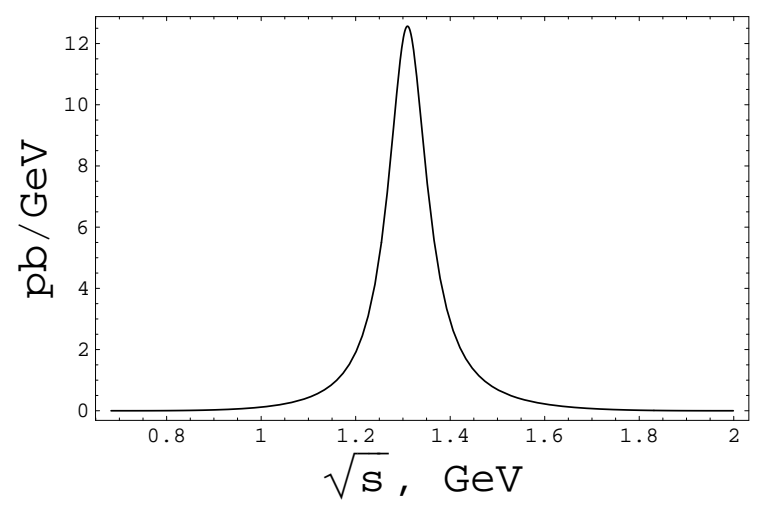

(b)

FIG. 3: a) The cutted $\pi^{0} \pi^{0}$ invariant mass spectra in the reaction $e^{+} e^{-} \rightarrow \pi^{0} \pi^{0} \gamma$ for $g_{f_{2} \rho^{\prime \prime} \rho^{\prime \prime}}=$ $g_{f_{2} \rho^{\prime} \rho^{\prime}}=g_{f_{2} \rho \rho}$ at $\sqrt{s}=2 \mathrm{GeV}$. The cutting is $100 \mathrm{MeV}$ around the $\omega$ mass in $\pi^{0}(1) \gamma$ and $\pi^{0}(2) \gamma$ invariant masses. The solid line is the sum of the cutted signal (long-dashed line), cutted $\omega \pi^{0}$ background (short-dashed line) and the interference (dashed line). b) The $\eta \pi^{0}$ invariant mass spectra in the reaction $e^{+} e^{-} \rightarrow \eta \pi^{0} \gamma$ at $\sqrt{s}=2 \mathrm{GeV}$ for $g_{a_{2} \omega^{\prime \prime} \rho^{\prime \prime}}=g_{a_{2} \omega^{\prime} \rho^{\prime}}=g_{a_{2} \omega \rho}$.

Using the $\Gamma\left(f_{2} \rightarrow \pi^{0} \pi^{0}, m\right)$ and the inverse propagator $D_{f_{2}}(m)$ from the Appendix, one gets Fig. [3a, where cutting $\pm 100 \mathrm{MeV}$ around $m_{\omega}$ in the $\pi^{0} \gamma$ mass was done to reduce the $\omega \pi^{0}$ background, see also Sec. IV.

The photon angular distribution is

$$
\begin{gathered}
\frac{d n_{\gamma}^{T}}{d \Omega}=\frac{T}{N} \\
T=\frac{\left(6 m_{f}^{4}+s^{2}\right)\left(1+\cos ^{2} \theta\right)+6 s m_{f}^{2} \sin ^{2} \theta}{3 m_{f}^{4}} \\
N=\frac{16 \pi}{9}\left(\frac{s^{2}}{m_{f_{2}}^{4}}+3 \frac{s}{m_{f_{2}}^{2}}+6\right)
\end{gathered}
$$

Table I. Elements of the $f_{2}$ spin density matrix, see Figs. (415)

\begin{tabular}{|c|c|c|c|c|c|}
\hline$\rho_{22}$ & $\frac{1}{T}\left(1+\cos ^{2} \theta\right)$ & $\rho_{21}$ & $\frac{1}{T} \frac{\sqrt{s} \sin (2 \theta)}{2 m_{f}}$ & $\rho_{20}$ & $-\frac{1}{T} \frac{s \sin ^{2} \theta}{\sqrt{6} m_{f}^{2}}$ \\
\hline$\rho_{11}$ & $\frac{1}{T} \frac{s \sin ^{2} \theta}{m_{f}^{2}}$ & $\rho_{10}$ & $\frac{1}{T} \frac{s^{3 / 2} \sin (2 \theta)}{2 \sqrt{6} m_{f}^{3}}$ & $\rho_{1,-1}$ & 0 \\
\hline$\rho_{00}$ & $\frac{1}{T} \frac{s^{2}\left(1+\cos ^{2} \theta\right)}{3 m_{f}^{4}}$ & $\rho_{2,-1}$ & 0 & $\rho_{2,-2}$ & 0 \\
\hline
\end{tabular}

The pion angular distribution in the rest frame of the tensor meson is

$$
W_{2}(\vartheta, \varphi)=\frac{15}{16 \pi}\left\{\sin ^{4} \vartheta \rho_{22}+\sin ^{2} 2 \vartheta \rho_{11}+3\left(\cos ^{2} \vartheta-\frac{1}{3}\right)^{2} \rho_{00}\right.
$$




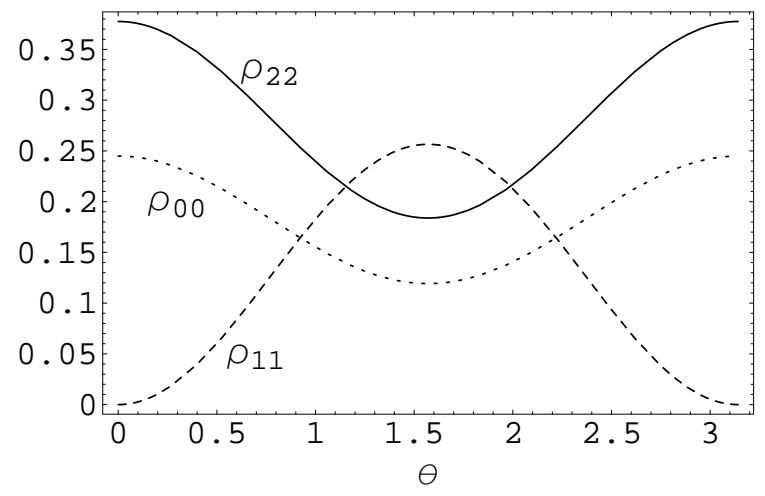

(a)

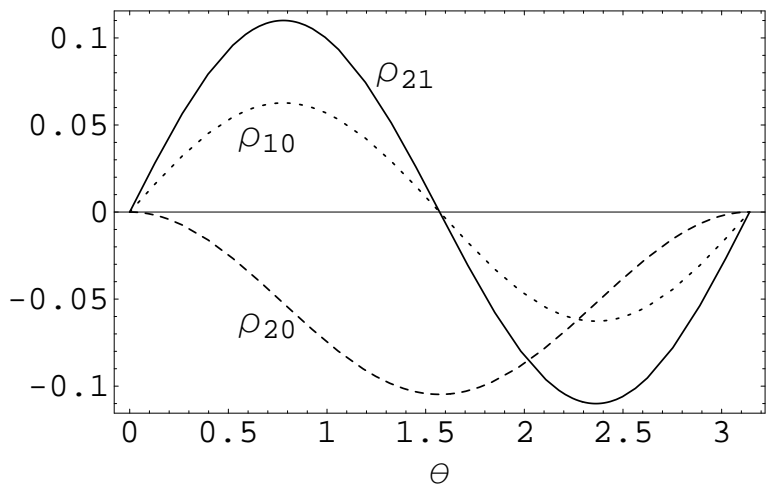

(b)

FIG. 4: The elements of the $f_{2}$ spin density matrix at $s=(1.5 \mathrm{GeV})^{2}$ (see Table I): a) $\rho_{22}$ (solid line), $\rho_{11}$ (dashed line), $\rho_{00}$ (short-dashed line); b) $\rho_{21}$ (solid line), $\rho_{20}$ (dashed line), $\rho_{10}$ (short-dashed line).

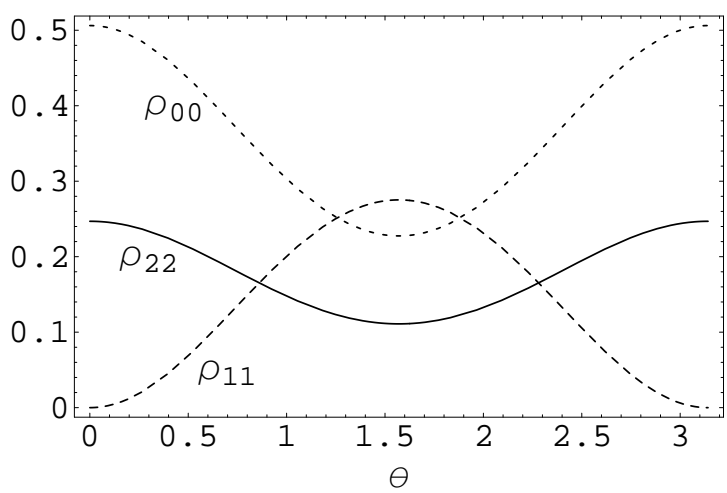

(a)

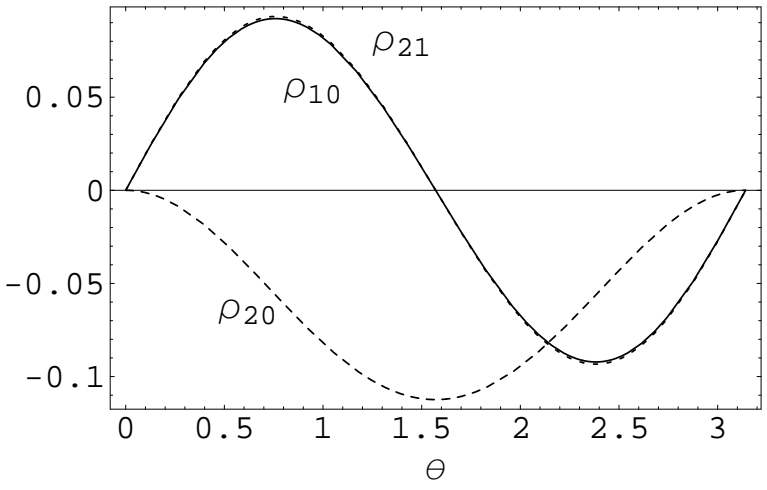

(b)

FIG. 5: The elements of the $f_{2}$ spin density matrix at $s=(2 \mathrm{GeV})^{2}$ (see Table I): a) $\rho_{22}$ (solid line), $\rho_{11}$ (dashed line), $\rho_{00}$ (short-dashed line); b) $\rho_{21}$ (solid line), $\rho_{20}$ (dashed line), $\rho_{10}$ (short-dashed line).

$$
\left.+2 \cos \varphi \sin 2 \vartheta\left(\sin ^{2} \vartheta \rho_{21}-\sqrt{6}\left(\cos ^{2} \vartheta-\frac{1}{3}\right) \rho_{10}\right)-2 \sqrt{6} \cos 2 \varphi \sin ^{2} \vartheta\left(\cos ^{2} \vartheta-\frac{1}{3}\right) \rho_{20}\right\}
$$

Here $\mathrm{z}$ axis is along the direction of the tenzor meson momentum in the $e^{+} e^{-}$s.c.m., $\mathrm{x}$ is in the reaction plane and $\mathrm{y}$ is perpendicular to the reaction plane [19, 20], $\vartheta$ is the polar angle and $\varphi$ is the azimuthal angle.

After integration over $\varphi$ one has 


$$
W_{2}(\vartheta)=\frac{15}{8}\left(\sin ^{4} \vartheta \rho_{22}+\sin ^{2} 2 \vartheta \rho_{11}+3\left(\cos ^{2} \vartheta-\frac{1}{3}\right)^{2} \rho_{00}\right)
$$

For the $e^{+} e^{-} \rightarrow a_{2} \gamma$ we may use Lagrangians similar to Eqs. (77), (8):

$$
L=g_{a_{2} \gamma \gamma} T_{\mu \nu} F_{\mu \sigma} F_{\nu \sigma}
$$

and

$$
L=g_{a_{2} V V^{\prime}} T_{\mu \nu} F_{\mu \sigma}^{V} F_{\nu \sigma}^{V^{\prime}},
$$

where $T_{\mu \nu}$ is the $a_{2}$ field, $V=\rho, \rho^{\prime}, \rho^{\prime \prime}$ and $V^{\prime}=\omega, \omega^{\prime}, \omega^{\prime \prime}$.

Analogically one may write $e^{+} e^{-} \rightarrow\left(\rho+\omega+\rho^{\prime}+\omega^{\prime}+\rho^{\prime \prime}+\omega^{\prime \prime}\right) \rightarrow a_{2}\left(\omega+\rho+\omega^{\prime}+\rho^{\prime}+\omega^{\prime \prime}+\rho^{\prime \prime}\right) \rightarrow$ $a_{2} \gamma$ cross section, neglecting $a_{2} \rho \omega^{\prime}, a_{2} \rho^{\prime} \omega$ and other cross vertices due to small overlap of the spatial wave functions of $\rho, \omega^{\prime}$ and $\rho^{\prime}, \omega$ and so on:

$$
\begin{gathered}
\sigma\left(e^{+} e^{-} \rightarrow a_{2} \gamma\right)=\frac{\pi^{2}}{9} \alpha^{3}\left(1-\frac{m_{a_{2}}^{2}}{s}\right)^{3}\left(\frac{s^{2}}{m_{a_{2}}^{4}}+3 \frac{s}{m_{a_{2}}^{2}}+6\right) \times \\
\left|\frac{m_{\rho}^{2} g_{a_{2} \rho \omega}}{f_{\rho} f_{\omega} D_{\rho}(s)}+\frac{m_{\omega}^{2} g_{a_{2} \rho \omega}}{f_{\rho} f_{\omega} D_{\omega}(s)}+\frac{m_{\rho^{\prime}}^{2} g_{a_{2} \rho^{\prime} \omega^{\prime}}}{f_{\rho^{\prime}} f_{\omega^{\prime}} D_{\rho^{\prime}}(s)}+\frac{m_{\omega^{\prime}}^{2} g_{a_{2} \rho^{\prime} \omega^{\prime}}}{f_{\rho^{\prime}} f_{\omega^{\prime}} D_{\omega^{\prime}}(s)}+\frac{m_{\rho^{\prime \prime}}^{2} g_{a_{2} \rho^{\prime \prime} \omega^{\prime \prime}}}{f_{\rho^{\prime \prime}} f_{\omega^{\prime \prime}} D_{\rho^{\prime \prime}}(s)}+\frac{m_{\omega^{\prime \prime}}^{2} g_{a_{2} \rho^{\prime \prime} \omega^{\prime \prime}}}{f_{\rho^{\prime \prime}} f_{\omega^{\prime \prime}} D_{\omega^{\prime \prime}}(s)}\right|^{2} \\
\Gamma\left(a_{2} \rightarrow \gamma \gamma\right)=\frac{\pi \alpha^{2}}{5}\left|\frac{g_{a_{2} \rho \omega}}{f_{\rho} f_{\omega}}+\frac{g_{a_{2} \rho^{\prime} \omega^{\prime}}}{f_{\rho^{\prime}} f_{\omega^{\prime}}}+\frac{g_{a_{2} \rho^{\prime \prime} \omega^{\prime \prime}}}{f_{\rho^{\prime \prime}} f_{\omega^{\prime \prime}}}\right|^{2} m_{a_{2}}^{3}=1.00 \pm 0.06 \mathrm{keV}[18] .
\end{gathered}
$$

Assuming $g_{a_{2} \rho^{\prime \prime} \omega^{\prime \prime}}=g_{a_{2} \rho^{\prime} \omega^{\prime}}=g_{a_{2} \rho \omega}$ gives Fig. 2b and $\eta \pi^{0}$ spectrum shown in Fig. 3b. The angular distributions are the same as Eqs. (16) and (17) (with $m_{f_{2}} \rightarrow m_{a_{2}}$ substitution in Eqs. (14) and (15)).

\section{THE BACKGROUND SITUATION}

Because of weakness of the signal cross-sections in the region $1.4 \div 2 \mathrm{GeV}$ it is needed to treat the background situation accurately. The $e^{+} e^{-} \rightarrow f_{0} \gamma \rightarrow \pi^{0} \pi^{0} \gamma$ and $e^{+} e^{-} \rightarrow a_{0} \gamma \rightarrow$ $\eta \pi^{0} \gamma$ are expected to be too small to be observed themselves, but for $e^{+} e^{-} \rightarrow f_{2} \gamma \rightarrow \pi^{0} \pi^{0} \gamma$ and $e^{+} e^{-} \rightarrow a_{2} \gamma \rightarrow \eta \pi^{0} \gamma$ it is possible to reduce background to the level much less than the signal one.

The background situation in case of $e^{+} e^{-} \rightarrow a_{0} \gamma \rightarrow \eta \pi^{0} \gamma$ and $e^{+} e^{-} \rightarrow a_{2} \gamma \rightarrow \eta \pi^{0} \gamma$ is rather friendly. The main background $e^{+} e^{-} \rightarrow \omega \pi^{0} \rightarrow \eta \pi^{0} \gamma$, obtained with the help of Ref. 


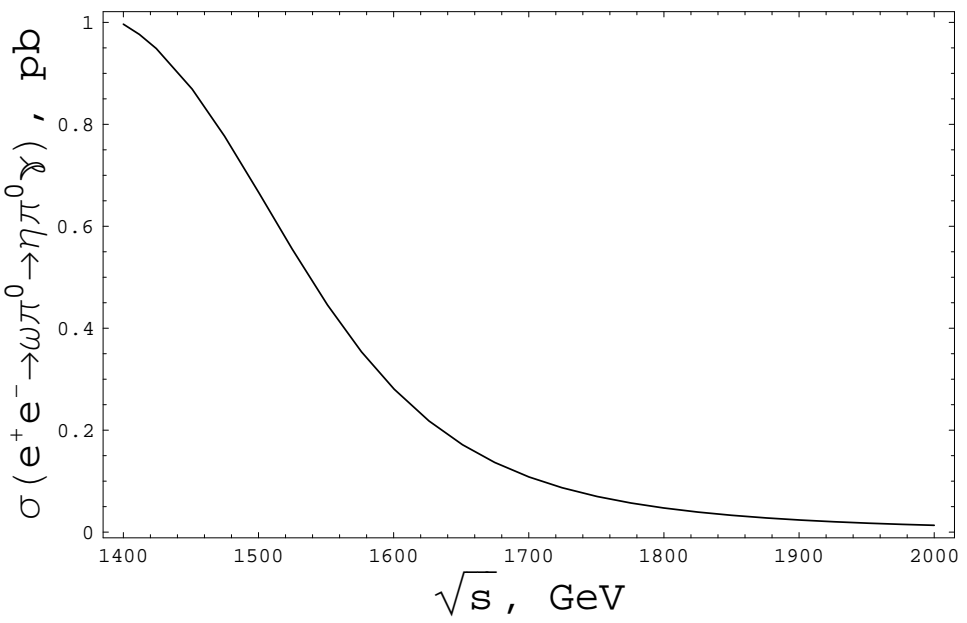

FIG. 6: The background cross-section $\sigma\left(e^{+} e^{-} \rightarrow \omega \pi^{0} \rightarrow \eta \pi^{0} \gamma\right)$, obtained with the help of Ref. [21].

[21], is much less than the signal cross-section, see Figs. 2b, 6. Other backgrounds are even much smaller.

The main background in case of $e^{+} e^{-} \rightarrow f_{0} \gamma \rightarrow \pi^{0} \pi^{0} \gamma$ and $e^{+} e^{-} \rightarrow f_{2} \gamma \rightarrow \pi^{0} \pi^{0} \gamma$ reactions comes from the $\omega \pi^{0}$ intermediate state also. Fig. 3a shows that cut $\pm 100 \mathrm{MeV}$ from the $\omega$ mass in the $\pi^{0} \gamma$ distribution reduces this background to the small level.

\section{CONCLUSION}

Our analysis shows that it should be possible to observe the reactions $e^{+} e^{-} \rightarrow a_{2} \gamma \rightarrow \eta \pi^{0} \gamma$ and $e^{+} e^{-} \rightarrow f_{2} \gamma \rightarrow \pi^{0} \pi^{0} \gamma$ at the energies near $2 \mathrm{GeV}$ in VEPP-2000 after reaching the project luminosity and probably in $D A \Phi N E$ after the planned full upgrade.

As to the reactions $e^{+} e^{-} \rightarrow a_{0} \gamma \rightarrow \eta \pi^{0} \gamma$ and $e^{+} e^{-} \rightarrow f_{0} \gamma \rightarrow \pi^{0} \pi^{0} \gamma$ at the energies $1.7-2$ $\mathrm{GeV}$, in the kaon loop model their cross sections are very small, while if the mere VDM (point-like $V S \gamma$ interaction) was correct they would be observed, see Fig. 1, Consequently, observation of tenzor contribution and non-observation of the scalar one would support the four quark nature of light scalar mesons [22 24] 


\section{ACKNOWLEDGEMENTS}

We thank A.A. Kozhevnikov very much for useful discussions concerning $e^{+} e^{-} \rightarrow K^{+} K^{-}$ cross-section, drawn in Ref. [14].

This work was supported in part by RFBR, Grant No. 13-02-00039, and Interdisciplinary Project No. 102 of the Siberian division of RAS.

\section{APPENDIX: THE PROPAGATORS OF THE $a_{2}(1320)$ AND $f_{2}(1270)$}

According to [25], we used the following inverse propagators for $T=a_{2}, f_{2}$ :

$$
D_{T}\left(m^{2}\right)=m_{T}^{2}-m^{2}-i m \Gamma_{T}(m),
$$

where

$$
\Gamma_{a_{2}}(m)=\Gamma\left(a_{2} \rightarrow \pi \pi, m\right)=\Gamma_{a_{2}}^{t o t} \frac{m_{a_{2}}^{2}}{m^{2}} \frac{p_{\eta \pi}^{5}(m)}{p_{\eta \pi}^{5}\left(m_{a_{2}}\right)} \frac{D_{2}\left(r_{a_{2}} p_{\eta \pi}\left(m_{a_{2}}\right)\right)}{D_{2}\left(r_{a_{2}} p_{\eta \pi}(m)\right)}
$$

and

$$
\Gamma_{f_{2}}(m)=\Gamma\left(f_{2} \rightarrow \pi \pi, m\right)+\Gamma\left(f_{2} \rightarrow K \bar{K}, m\right)+\Gamma\left(f_{2} \rightarrow 4 \pi, m\right)
$$

which is dominated by

$$
\Gamma\left(f_{2} \rightarrow \pi \pi, m\right)=\Gamma_{f_{2}}^{t o t} B\left(f_{2} \rightarrow \pi \pi\right) \frac{m_{f_{2}}^{2}}{m^{2}} \frac{p_{\pi \pi}^{5}(m)}{p_{\pi \pi}^{5}\left(m_{f_{2}}\right)} \frac{D_{2}\left(r_{f_{2}} p_{\pi \pi}\left(m_{f_{2}}\right)\right)}{D_{2}\left(r_{f_{2}} p_{\pi \pi}(m)\right)} .
$$

Here $D_{2}(x)=9+3 x^{2}+x^{4}\left[26\right.$ ], and we take from Ref. [25] $m_{a_{2}}=1322 \mathrm{MeV}, \Gamma_{a_{2}}^{t o t}=116$ $\mathrm{MeV}, r_{a_{2}}=1.9 \mathrm{GeV}^{-1}, m_{f_{2}}=1272 \mathrm{MeV}, \Gamma_{f_{2}}^{\text {tot }}=196 \mathrm{MeV}, B\left(f_{2} \rightarrow \pi \pi\right)=0.848, r_{f_{2}}=8.2$ $\mathrm{GeV}^{-1}$. The $\Gamma\left(f_{2} \rightarrow K \bar{K}, m\right)$ has the similar form as Eq. (25), we use $B\left(f_{2} \rightarrow K \bar{K}\right)=0.046$. The $\Gamma\left(f_{2} \rightarrow 4 \pi, m\right)$ may be approximated by the S-wave $f_{2} \rightarrow \rho \rho \rightarrow 4 \pi$ decay width as in Ref. [25], but for simplicity we used the dependence $\Gamma\left(f_{2} \rightarrow 4 \pi, m\right)=\Gamma\left(f_{2} \rightarrow 4 \pi, m_{f_{2}}\right) \frac{m^{2}}{m_{f_{2}}^{2}}$ as in Ref. [27], here $B\left(f_{2} \rightarrow 4 \pi\right)=0.106$.

[1] N.N. Achasov and V.N. Ivanchenko, Nucl. Phys. B315, 465 (1989).

[2] N.N. Achasov and V.V. Gubin, Phys. Rev. D56, 4084 (1997). 
[3] M.N. Achasov et al., Phys. Lett. B438, 441 (1998); M.N. Achasov et al., Phys. Lett. B440, 442 (1998); M.N. Achasov et al., Phys. Lett. B479, 53 (2000); M.N.Achasov et al., Phys. Lett. B485, 349 (2000).

[4] R.R. Akhmetshin et al., Phys. Lett. B462, 380 (1999).

[5] A.Aloisio et al., Phys. Lett. B536 (2002).

[6] A.Aloisio et al., Phys. Lett. B537 (2002); C. Bini, P. Gauzzi, S. Giovanella, D. Leone, and S. Miscetti, KlOE Note 173 06/02, http://www.lnf.infn.it/kloe/.

[7] N.N. Achasov, V.V. Gubin, Phys. Rev. D63, 094007 (2001).

[8] N.N. Achasov, Nucl. Phys. A 728, 425 (2003).

[9] N.N. Achasov and A.V. Kiselev, Phys. Rev. D68, 014006 (2003).

[10] N.N. Achasov and A.V. Kiselev, Phys. Rev. D73, 054029 (2006); Erratum-ibid. D74, 059902 (2006); Yad. Fiz. 70, 2005 (2007) [Phys. At. Nucl. 70, 1956 (2007)].

[11] N.N. Achasov and A.V. Kiselev, Phys. Rev. D83, 054008 (2011); Phys. Rev. D85, 094016 (2012).

[12] P. M. Ivanov et al. Phys. Lett. 107B, 297 (1981).

[13] D. Bisello et al. Z. Phys. C39, 13 (1988).

[14] N.N. Achasov, A.A. Kozhevnikov, Phys. Rev. D57, 4334 (1998).

[15] The mere VDM prediction is obtained by the removement of the loop integral $\bar{g}(m)$ and normalization of the cross-section on the signal one Eqs. (11), (2) at $\sqrt{s}=m_{\phi}$.

[16] N.N. Achasov, Phys. Lett. B22, 139 (1989).

[17] N.N. Achasov and V.A. Karnakov, Z. Phys. C30, 141 (1986).

[18] J. Beringer et al. (Particle Data Group), Phys. Rev. D86, 010001 (2012).

[19] K. Gottfried and J. Jackson, Nuovo Cim. 33, 3589 (1964).

[20] J.Jackson, Nuovo Com. 34, 6692 (1964).

[21] N.N. Achasov, A.A. Kozhevnikov, Phys. Rev. D55, 2663 (1997).

[22] R.L. Jaffe, Phys. Rev. D15, 267 (1977).

[23] R.L. Jaffe, Phys. Rev. D15, 281 (1977).

[24] N.N. Achasov, S.A. Devyanin and G.N. Shestakov, Z. Phys. C22, 53 (1984);

[25] N.N. Achasov and G.N. Shestakov, Physics-Uspekhi 54, 799 (2011) [Usp. Fiz. Nauk. 181, 827 (2011)].

[26] J.M. Blatt and V.F. Weisskopf, Theoretical Nuclear Physics (Wiley, New York, 1952), pp. 
359-365 and 386-389.

[27] S. Uehara et al, Phys. Rev. D82, 114031 (2010). 